
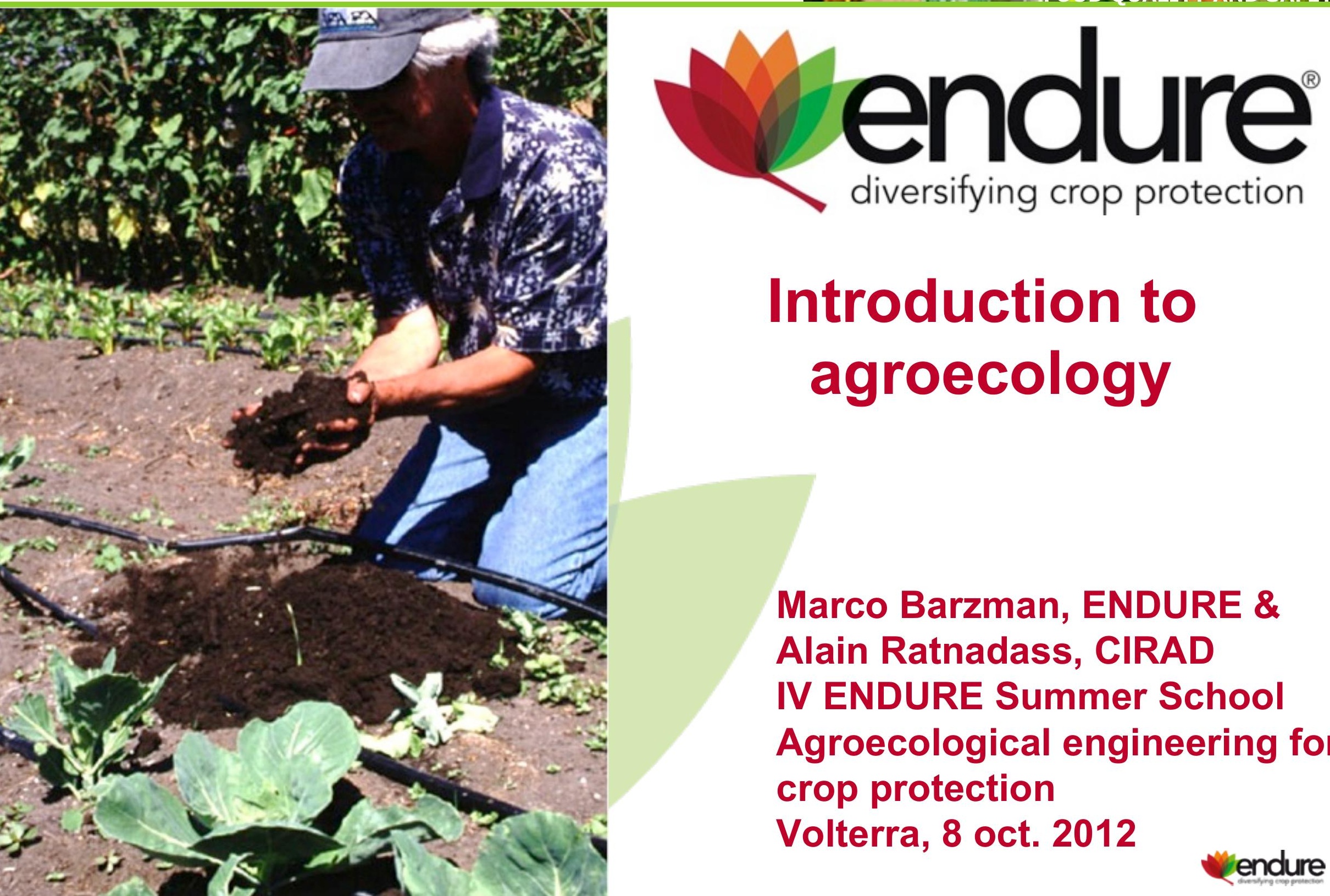

\title{
Introduction to agroecology
}

Marco Barzman, ENDURE \& Alain Ratnadass, CIRAD IV ENDURE Summer School Agroecological engineering for crop protection Volterra, 8 oct. 2012 


\section{Origins}

* Latin America: 1970s - 1980s - difficult political situations

* Nth America: environmental protection, think globally act locally

* United Nations Conference on Environment and Development, Rio Summit 1992

* Reaction to green revolution 


\section{Assumptions...}

\section{* Population bomb, Paul Erlich, 1968}

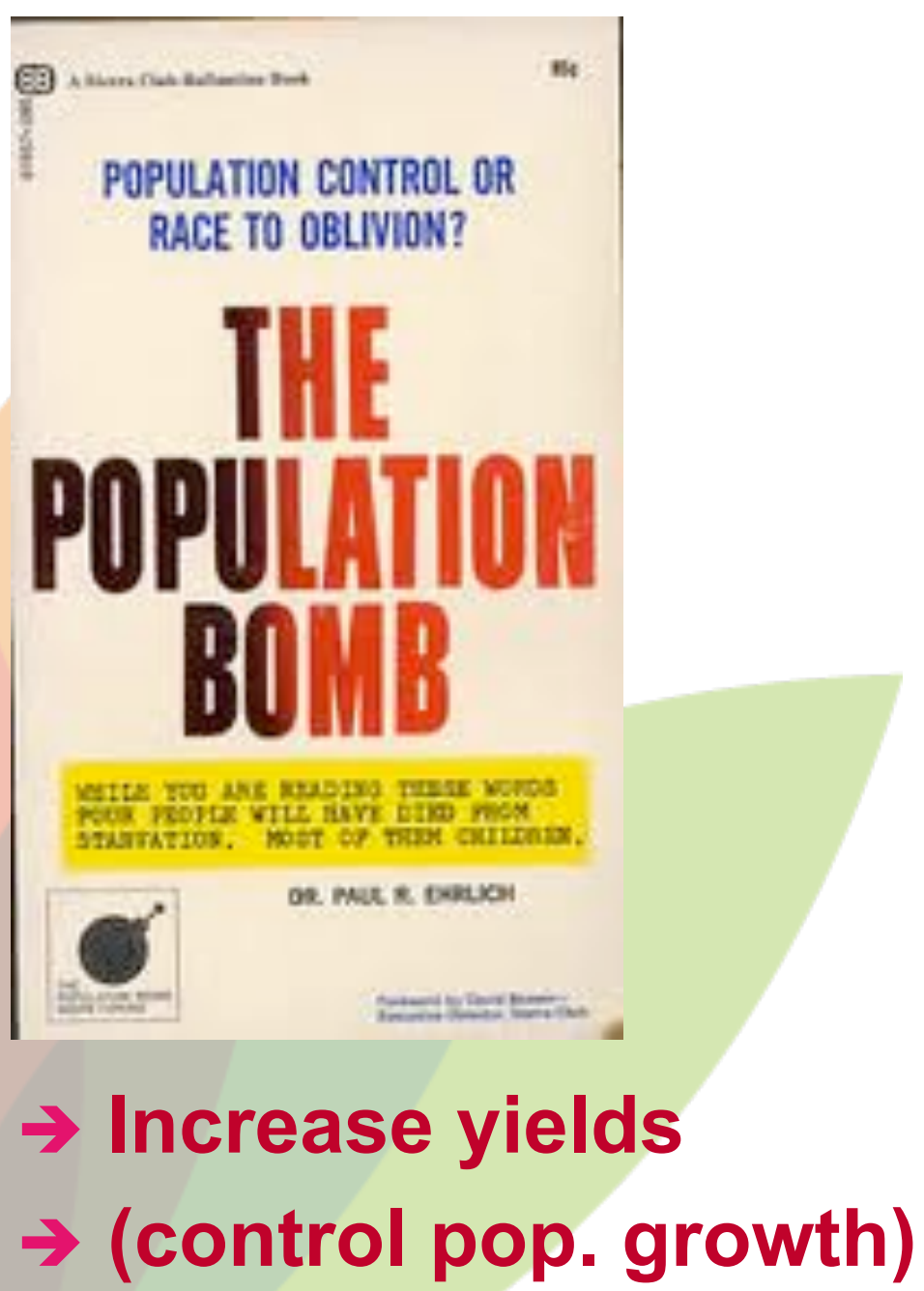


* 12 Myths, Frances Moore-Lappé, 1986

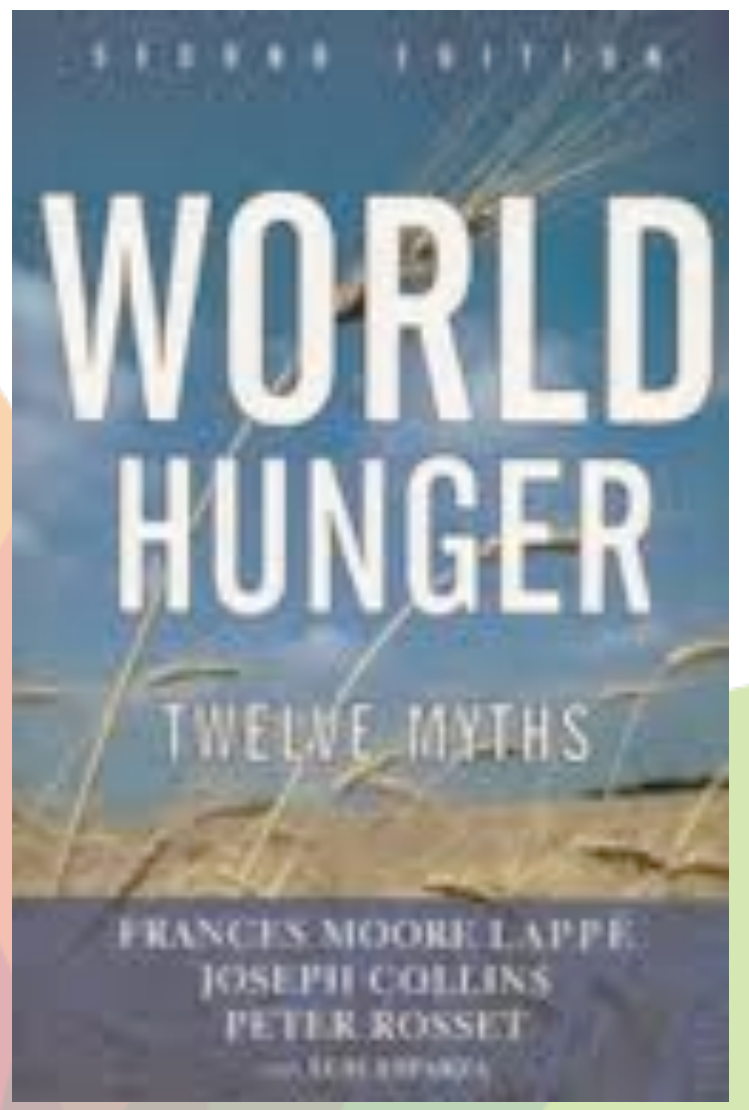

$\Rightarrow$ Improve access to production 


\section{Doubly green revolution}
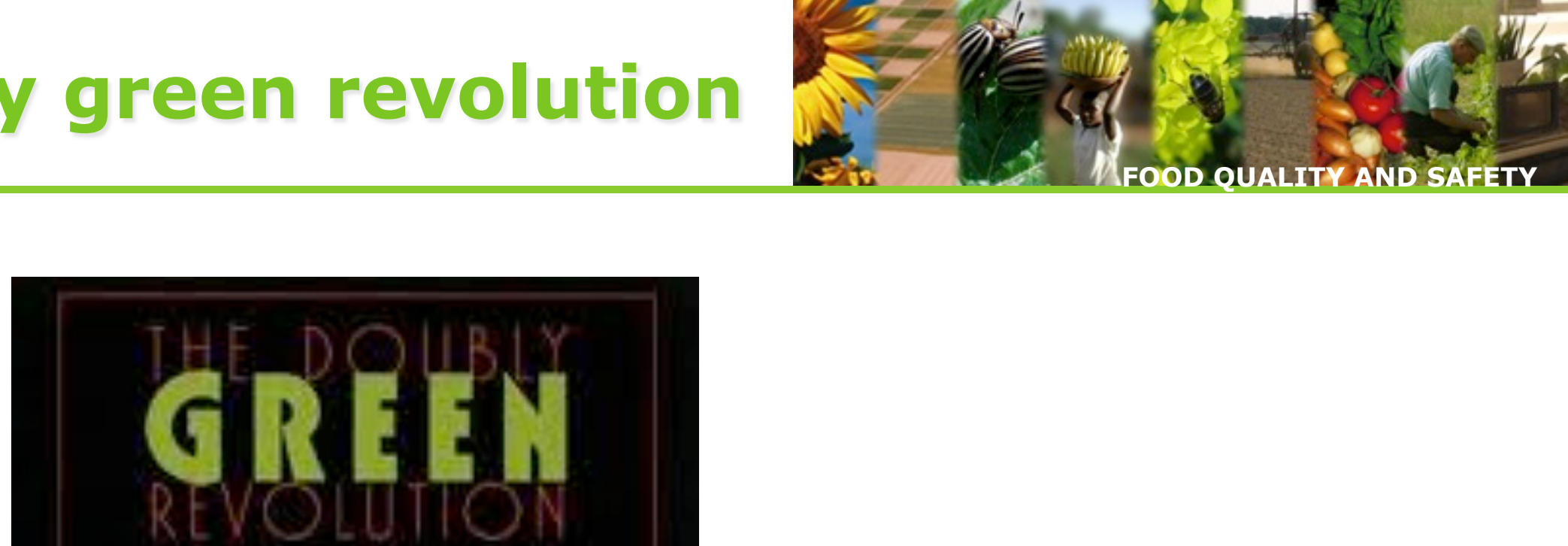

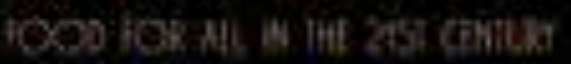
GORDOH CONWAY

and now $\rightarrow$

"evergreen revolution" 


\section{Critique}

\section{GREEN REVOLUTION}

Curse or Blessing?
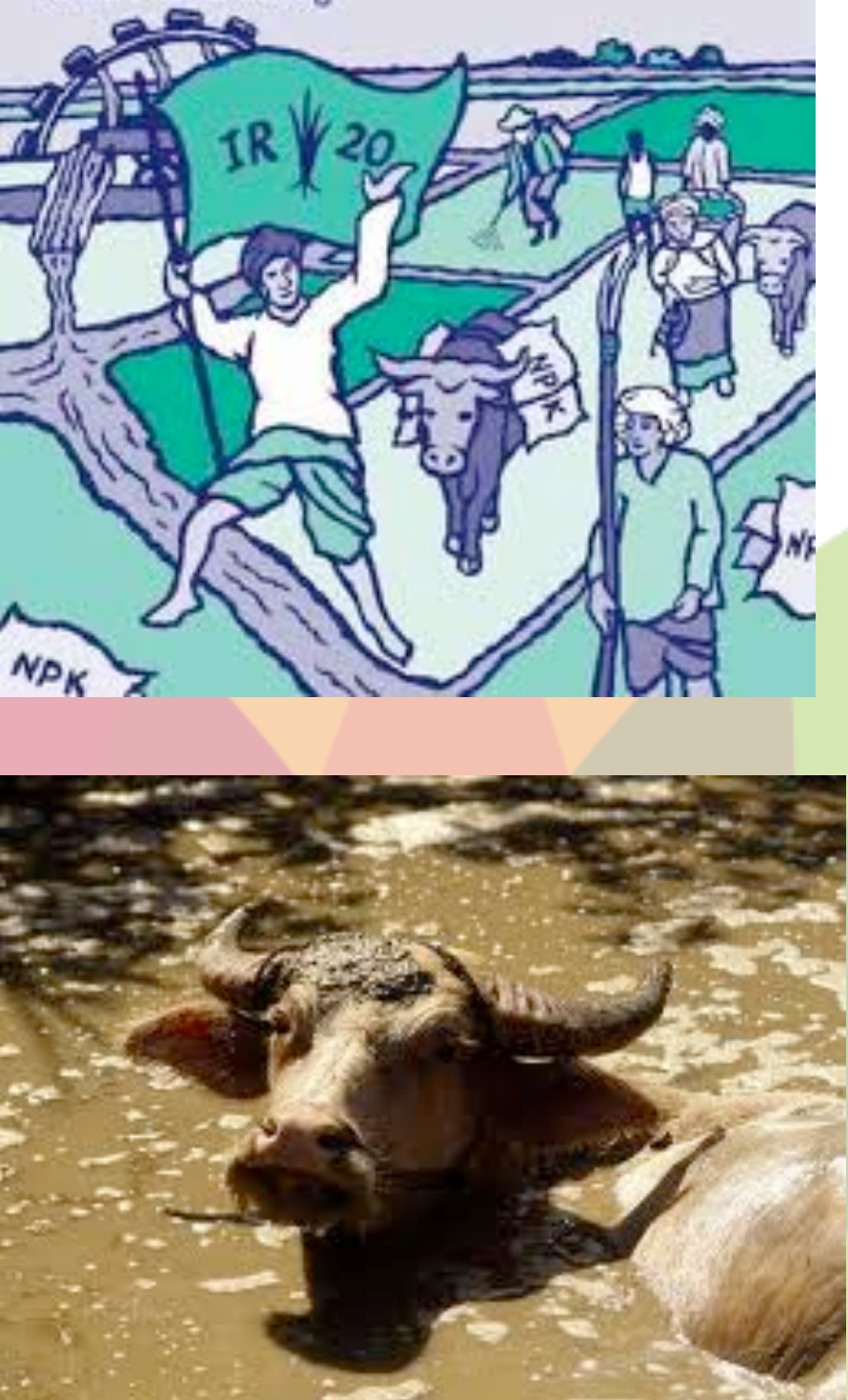

* Social justice - unequal distribution of benefits

* Technology \& dependence (machinery, seed, NPK, loans, markets, ...)

* Knowledge - expert K vs. IKS

* Appropriateness of Northern model applied to unknown system

- Unexpected consequences

- Diet (e.g. rice in Bangladesh)

- Public health (e.g. water buffalo)

- Biodiversity

* Local resources, local systems 


\section{Critique}

\section{Local rice variety use among project farmers}

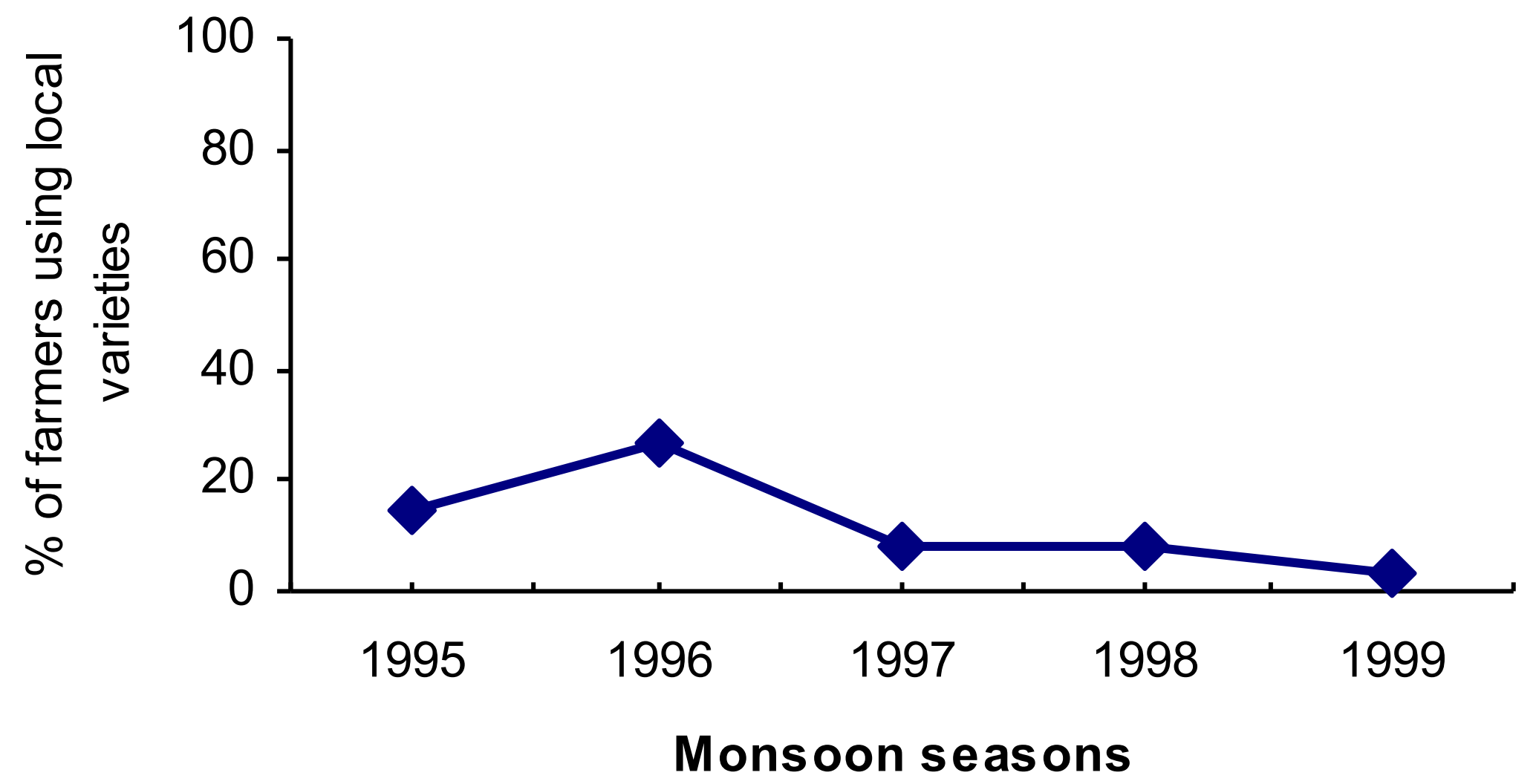


* Genetic erosion - in-situ conservation

* Biopiracy - address rights of traditional communities

* Social justice - land reform

* Indigenous Knowledge Systems I understanding traditional systems

* Autonomy / food sovereignty

- Appropriate technology 


\section{IKS: weaver ant in VN}
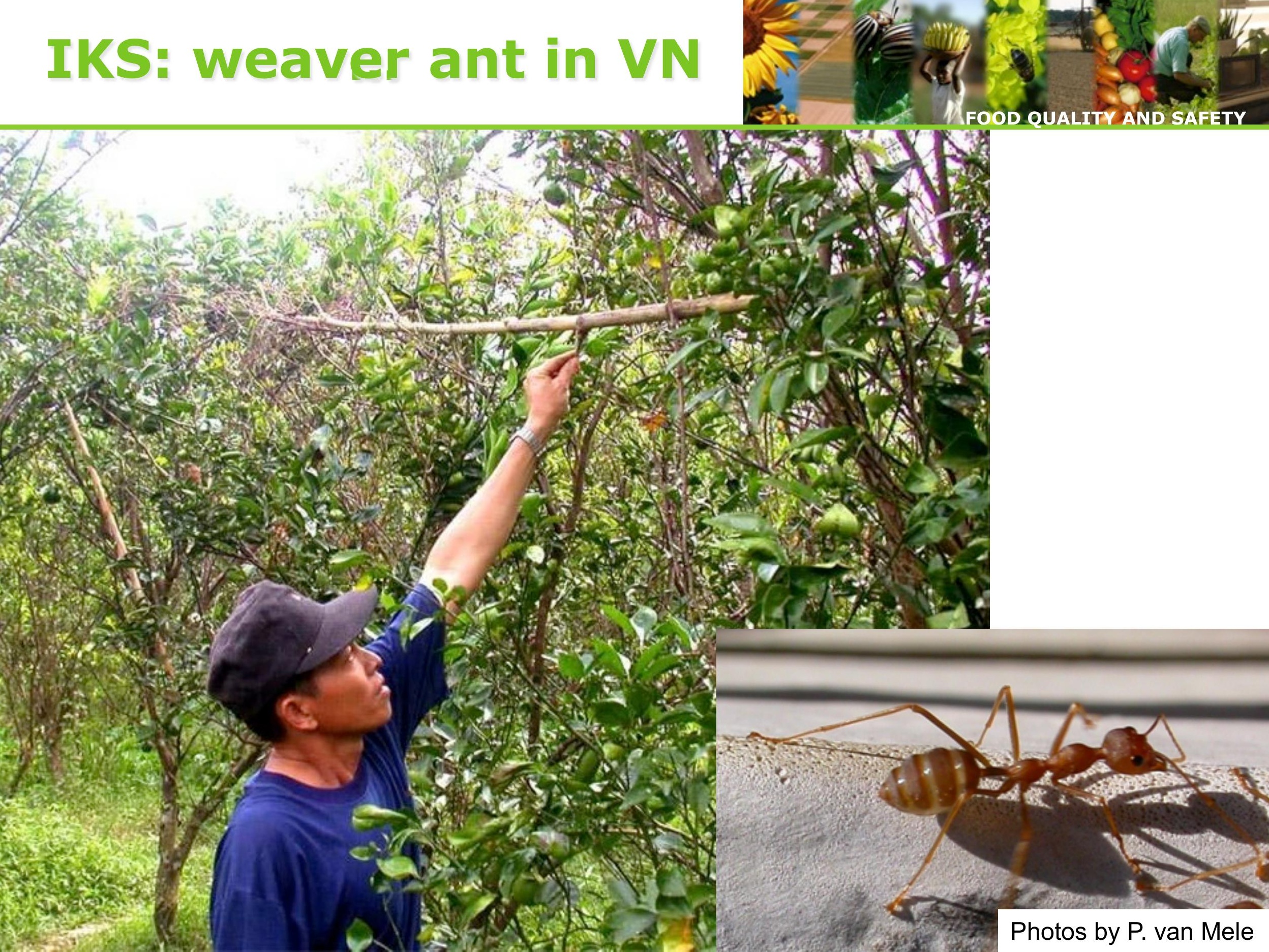
IKS: weaver ant in VN

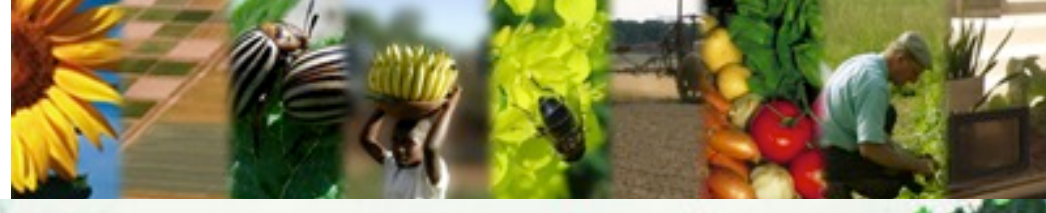

20

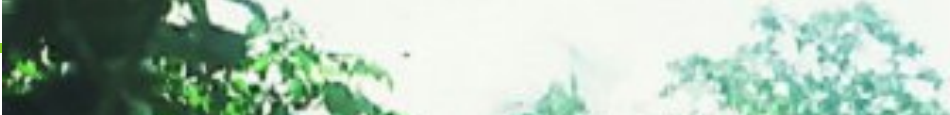

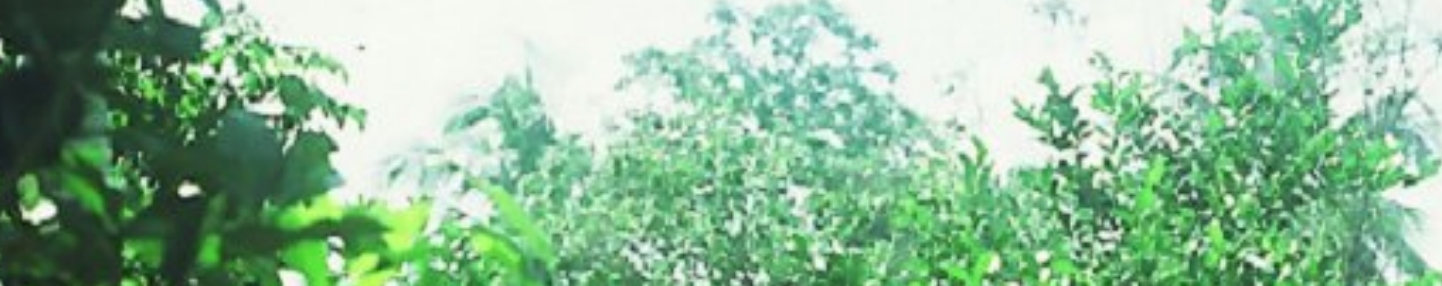

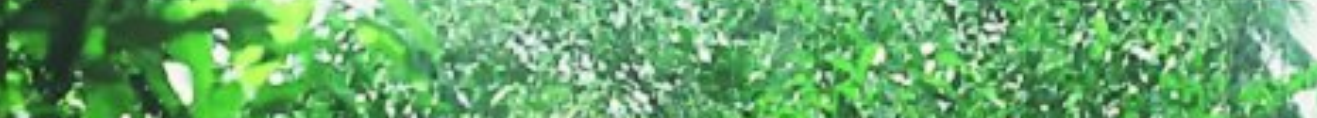

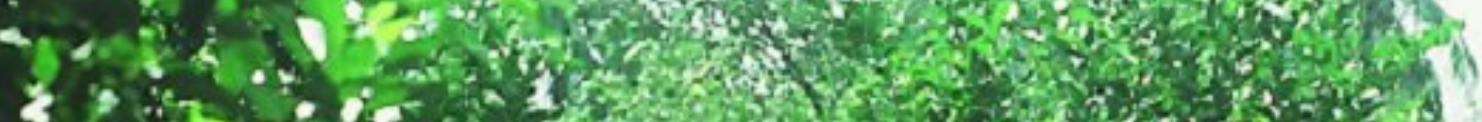

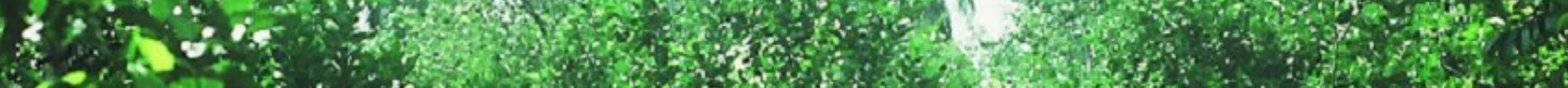

(3)

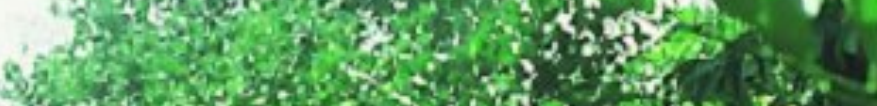

4050 .

The

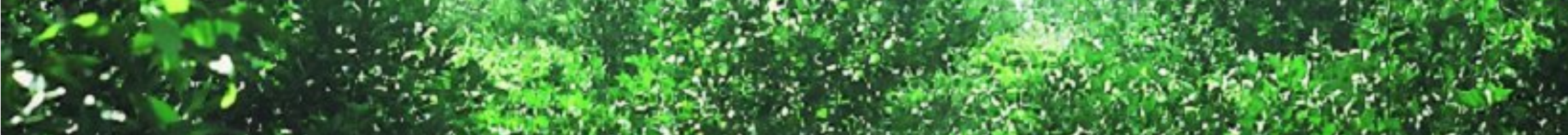

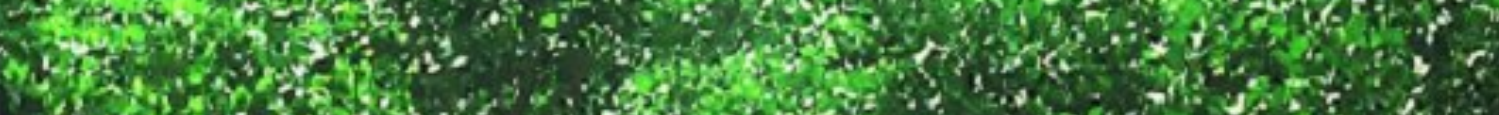

(15)

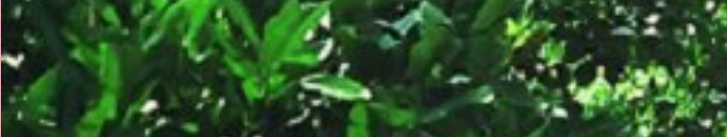

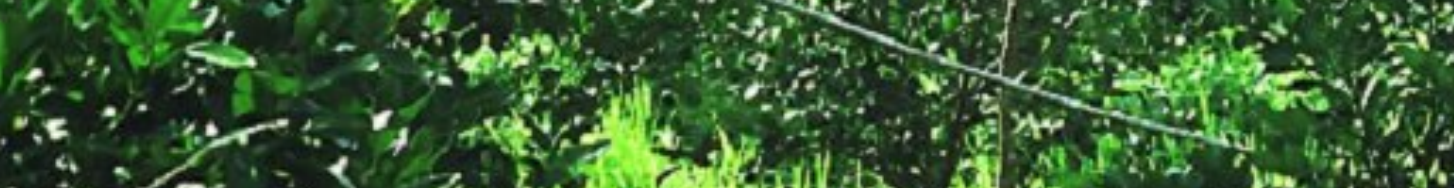

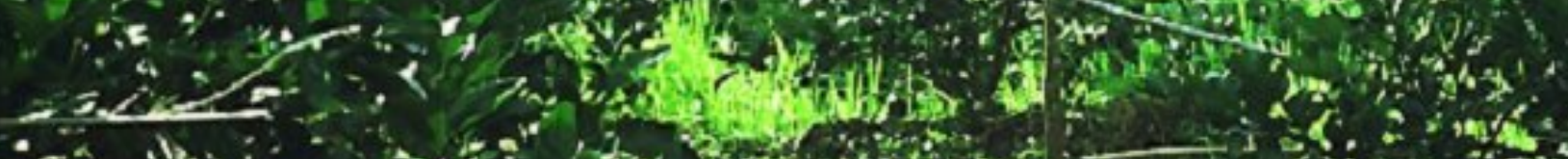

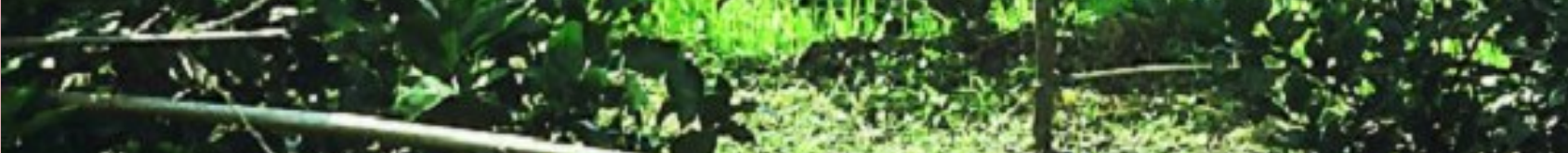

-

Photo by M. Barman

Photo by M. Barzman

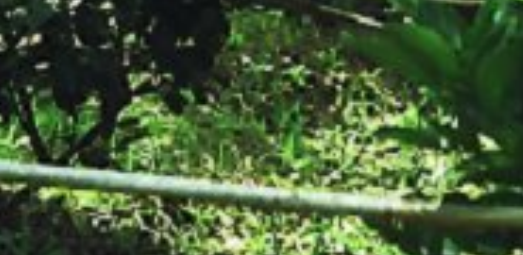

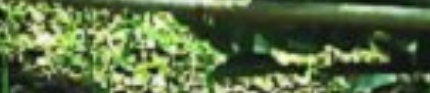

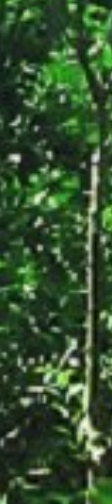

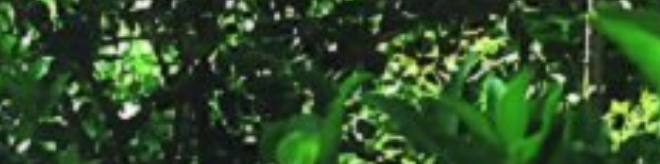

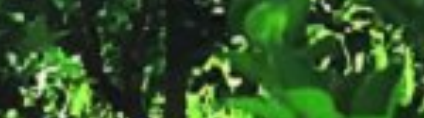




\section{IKS: weaver ant in VN}

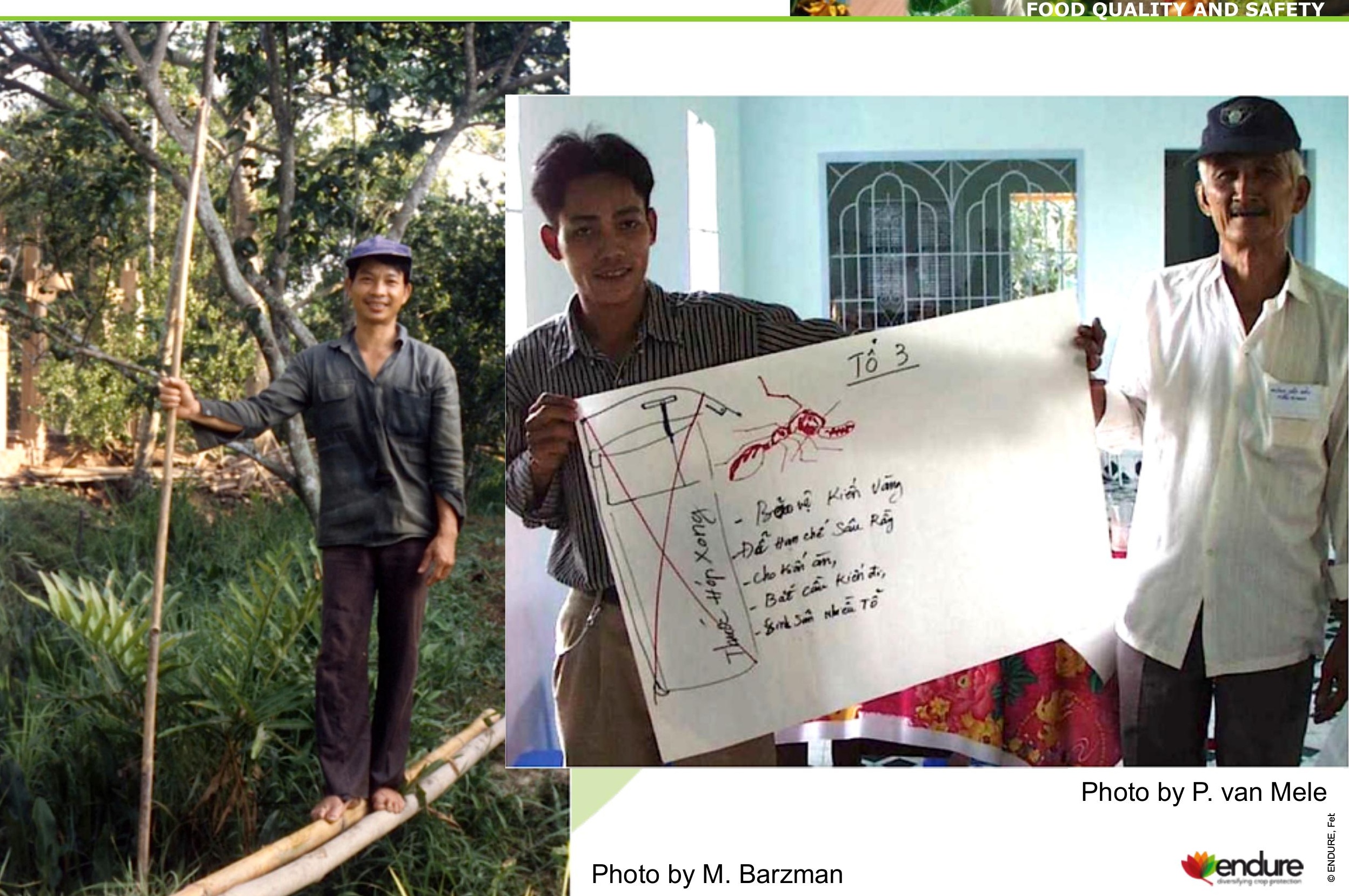




\section{Some of the people...}
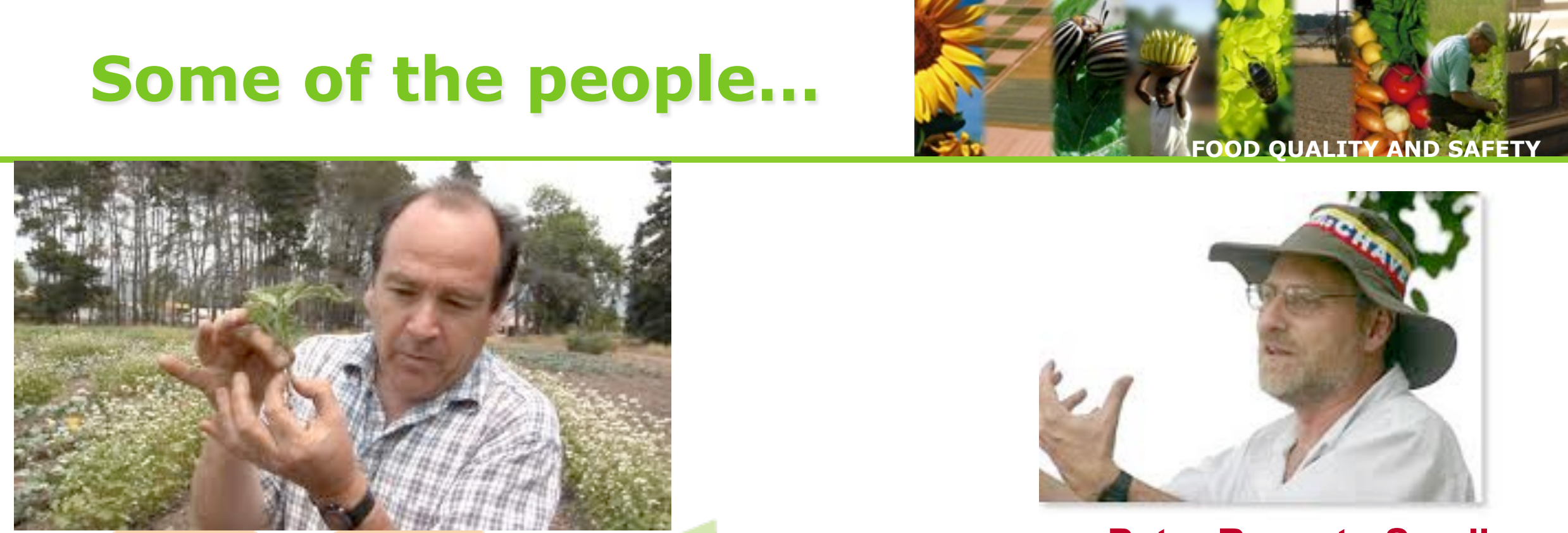

* Miguel Altieri - Agroecology: the science of sustainable agriculture, 1986

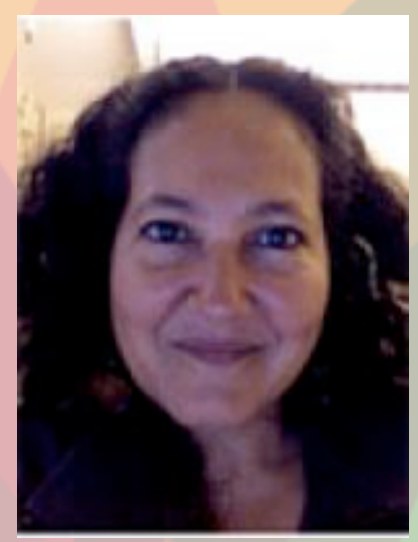

* Ivette Perfecto, biodiversity in ag

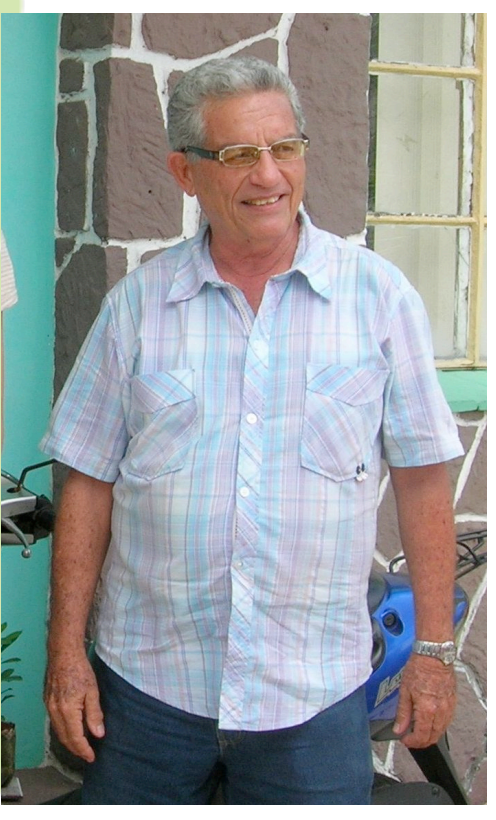

* Fernando Funes, Cuba

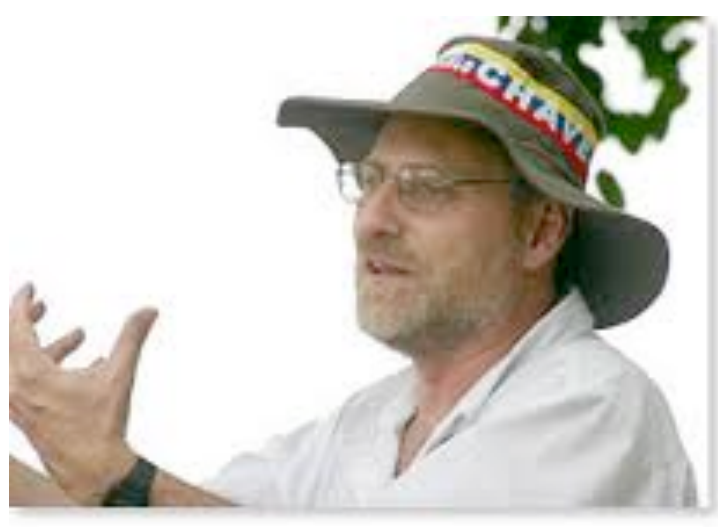

* Peter Rosset - Small Is Bountiful, 1999 


\section{Strategies}

* Anti-GMO movement

Peace

Compost on the First of Spping $3 / 30 / 02$ Quet2ol Rodrigo Lisa. Laura greens. manure,
* Locavore: Food-shed, CSAs, Farmers' markets, U pick, agroecotourism

* School programmes - school gardens, organic lunch, salad bar

* Intentional communities

* Farm worker rights

* Urban ag

* Organic ag 


\section{New directions for ag}

* Wildlife-friendly agriculture

* Native spp.

- Low-input organic farming

* Permaculture

* Biodynamic

* French intensive horticulture

* Urban agriculture

* Crop-livestock integration

* Agroforestry

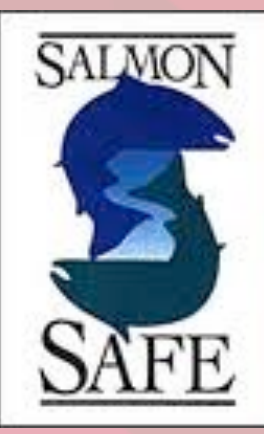

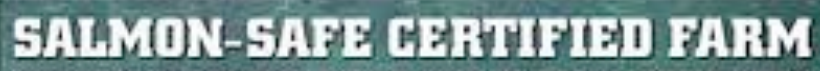
WOAKIN TO PROTEGT WATYUE FISH DN MARW WATERASHEDS
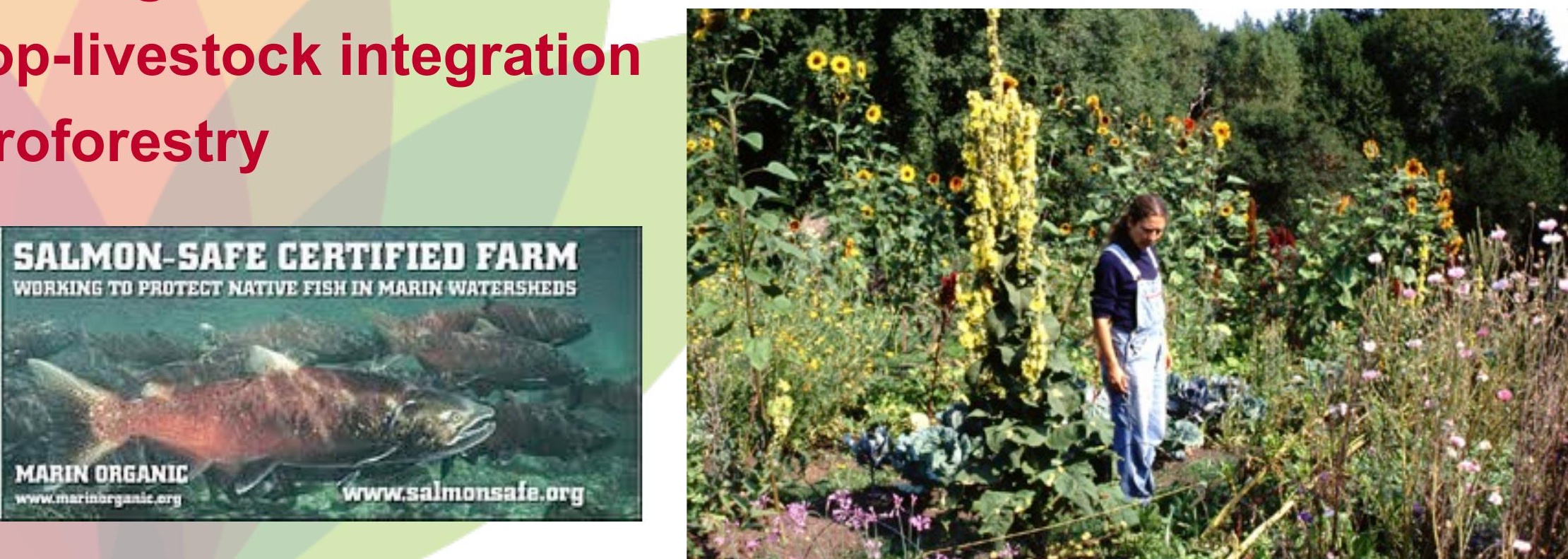


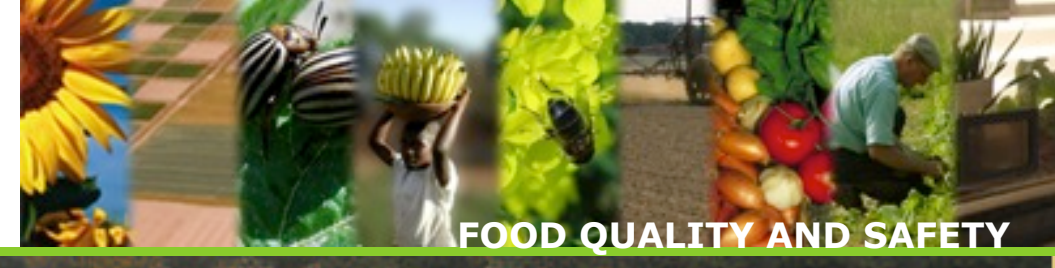

\section{OCCIDENTAL ARTS \& ECOLOGY CENTER}

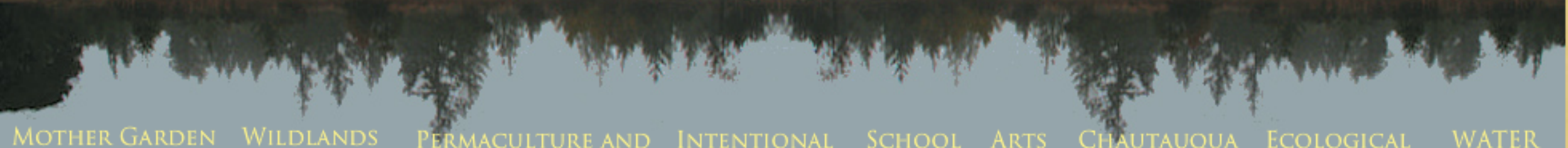
BIODIVERSITY BIODIVERSITY ECOLOGICAL DESIGN COMMUNITIES GARDENS ARTITUTE

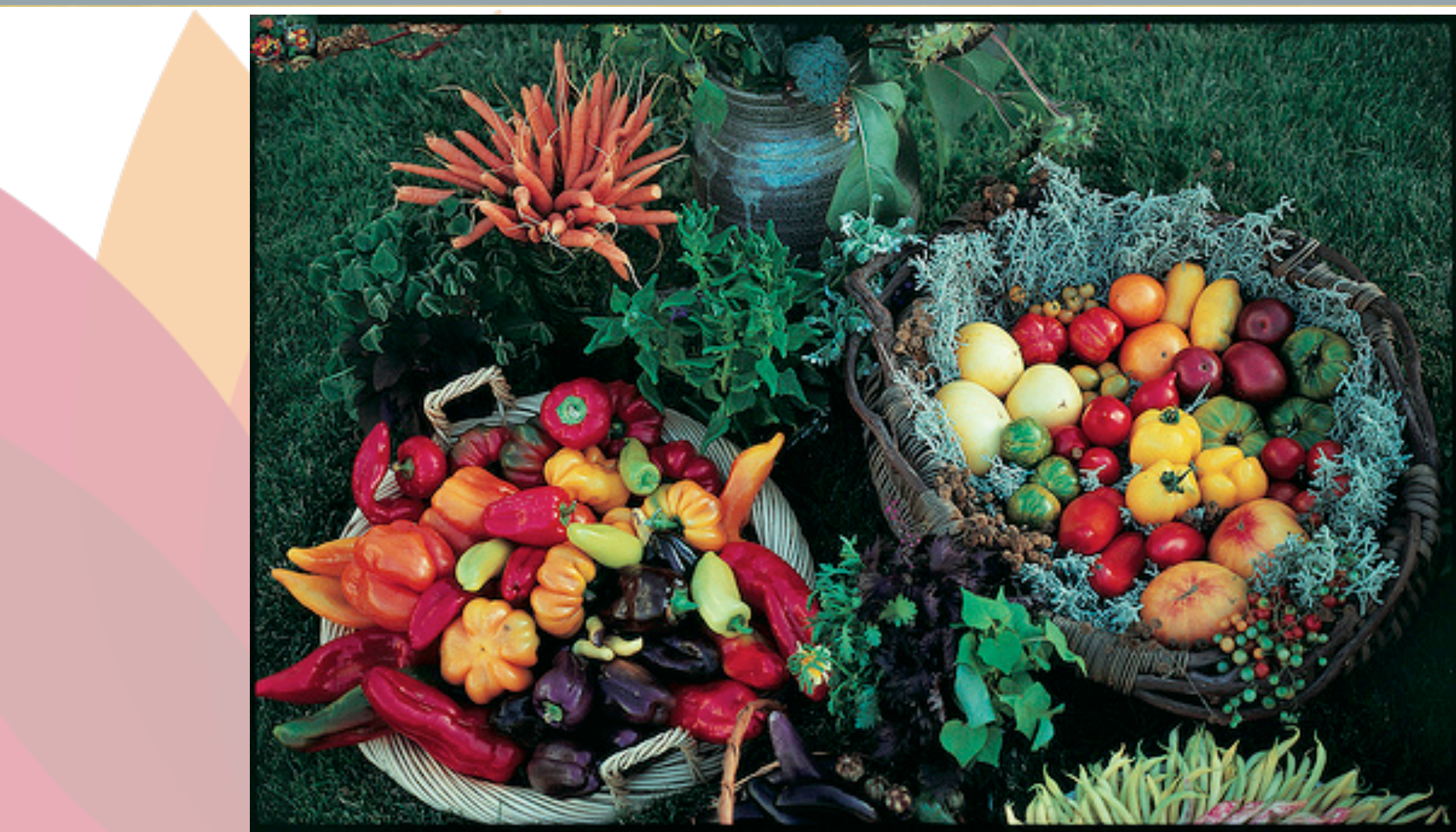

Wendure

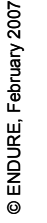




\section{What is agroecology?}

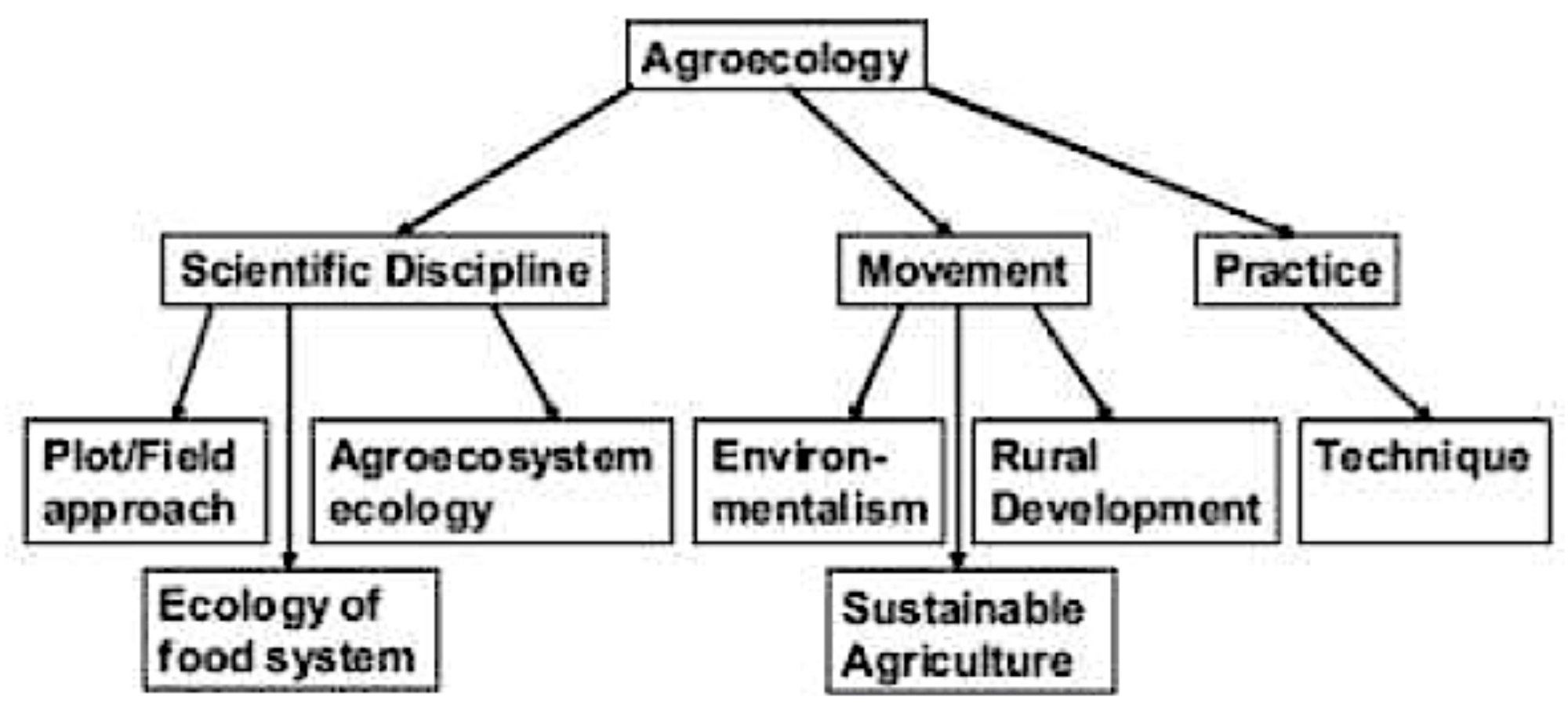

From Wezel et al, 2009 
- Agroecology as a scientific approach?

- Holistic vs. reductionist approach

- Systems sciences

- Multi-disciplinary approaches ex. NMJ weed sci $\rightarrow$ farm management $Q$

- Multi-sector - who is the expert?

-Ecology - from "contemplative Ecology" of 'natural' systems to management of agroecosystems

- Ethnobotany / understanding traditional systems

- Descriptive agroecology on developed country agriculture?

- Ecosystem services (multi-functional agriculture) 
The application of ecological concepts and principles to the design and management of sustainable agroecosystems 


\section{Sustainability in ag}

* Minimal impact on environment

* Preserve \& rebuild soil fertility

* Use water efficiently

* Rely on internal resources \& nutrient cycling

* Conserve biological diversity

- Enable local control of agricultural resources \& access to knowledge 
Relying on ecosystem processes

* Energy flow

* Nutrient cycling

- Soil building

* Succession

* Disturbance

* Interactions between species

* Abiotic/biotic interactions 


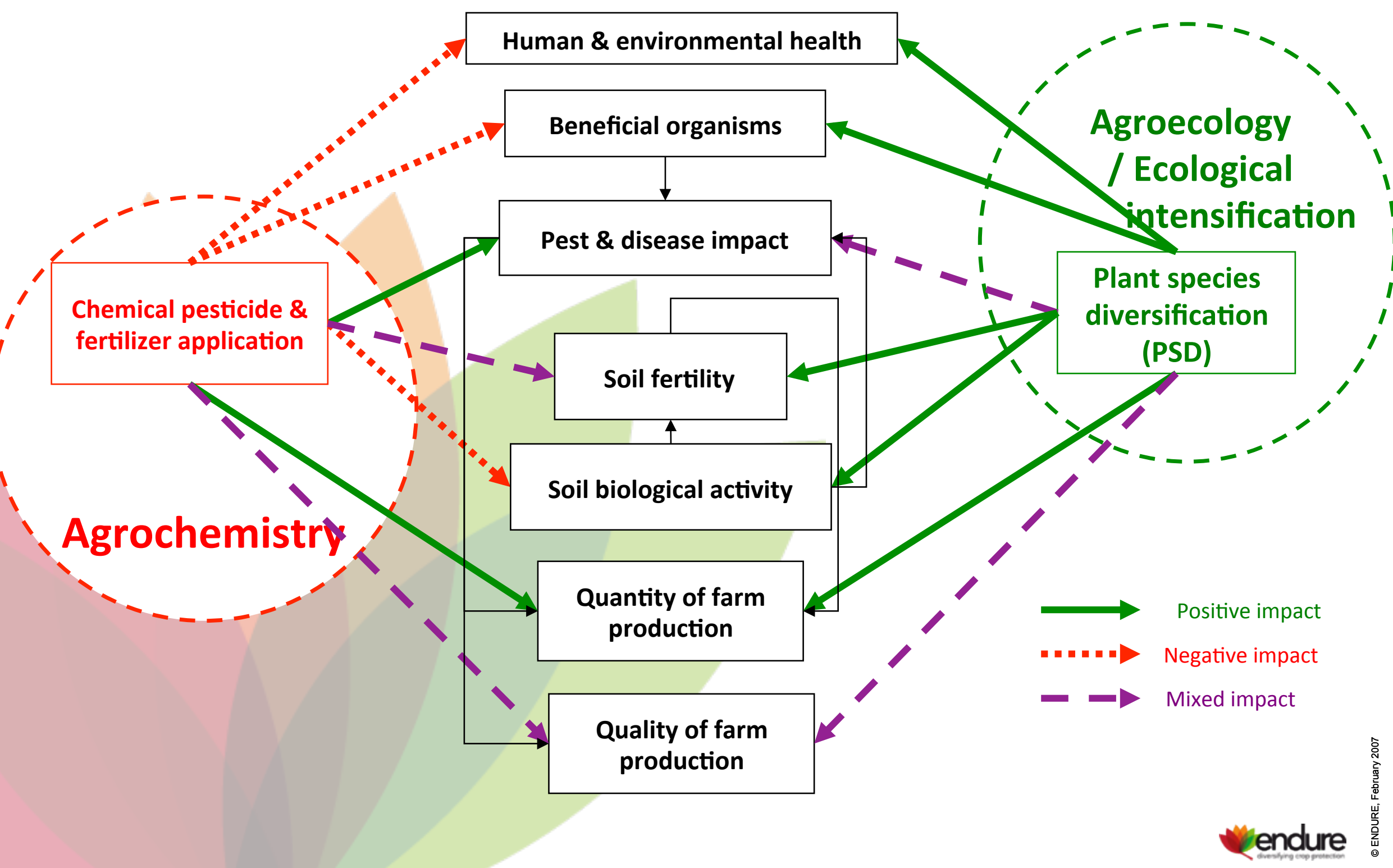




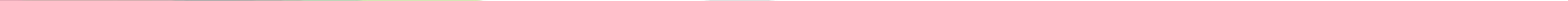





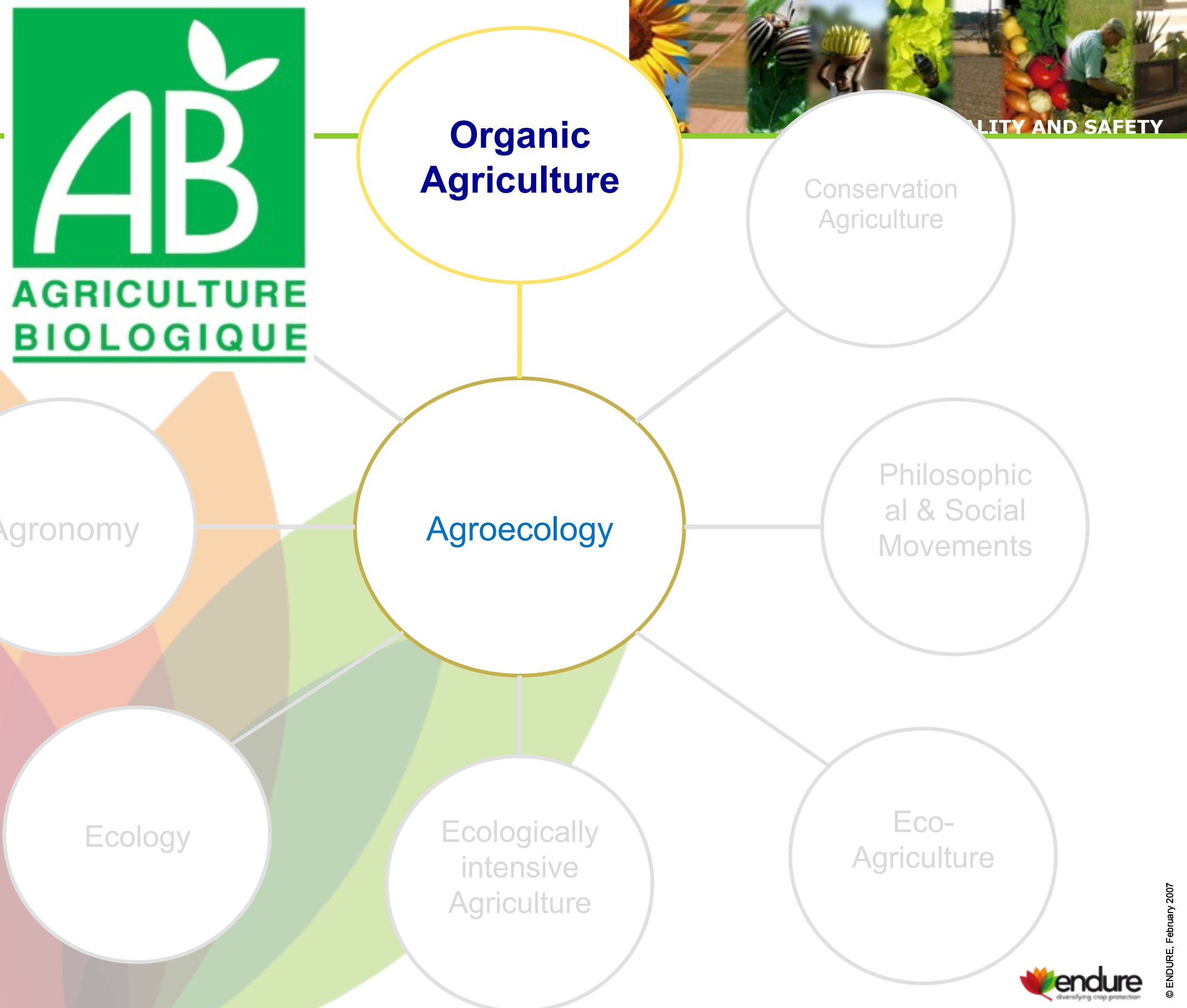





\section{PIERRE RABHI}

\section{MANIFESTE \\ POUR}

LA TERRE ET

L'HUMANISME

preface de Nicolas Hulot

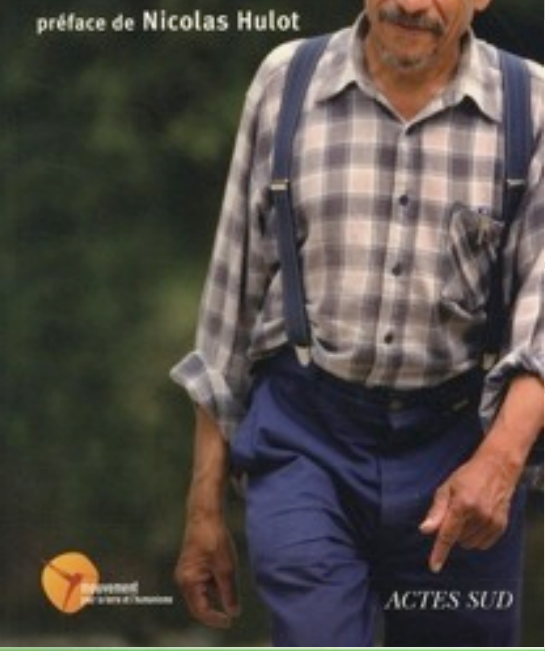

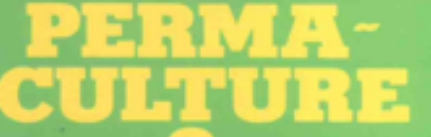
20

Aménagements pratiques

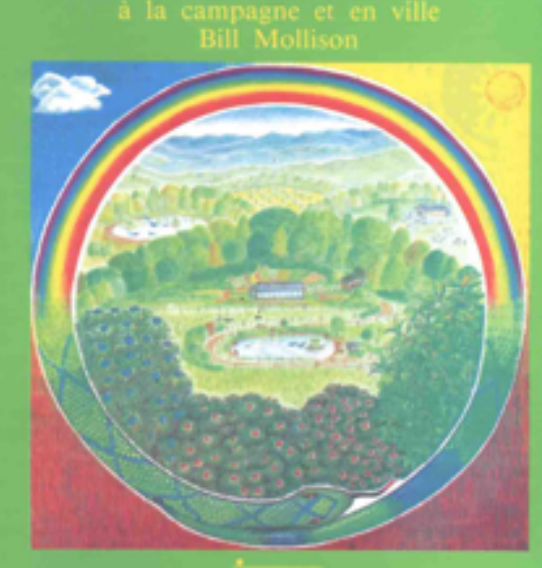

The Journal of Peasant Studies

Vol. 38, No. 3, July 2011, 587-612

The agroecological revolution in Latin America: rescuing nature, ensuring food sovereignty and empowering peasants

Miguel A. Altieri and Victor Manuel Toledo

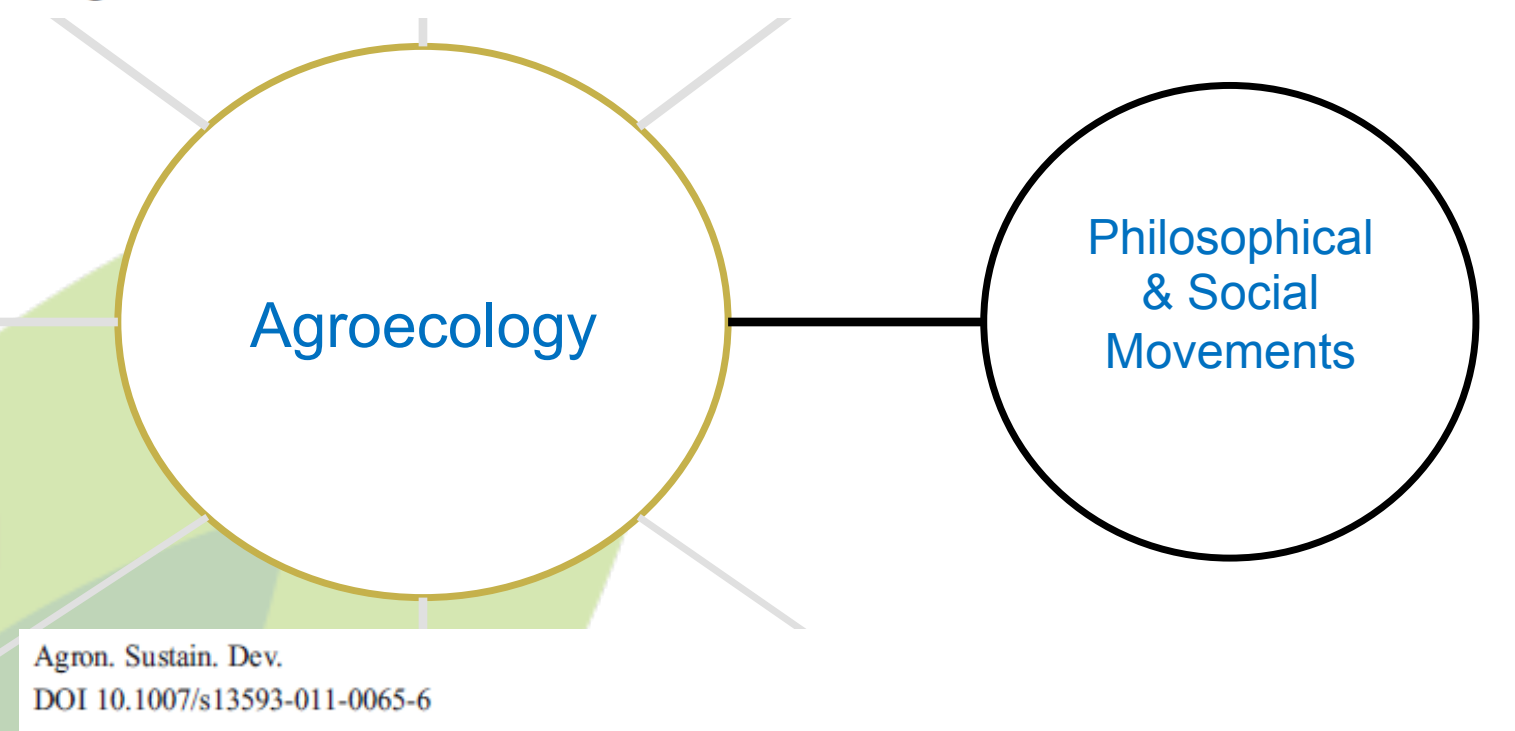

REVIEW ARTICLE

Agroecologically efficient agricultural systems for smallholder farmers: contributions to food sovereignty

Miguel A. Altieri • Fernando R. Funes-Monzote •

Paulo Petersen 
RESPONSE TO ALTIERI AND FARVAR ARTICLES

Biodiversity Conservation in Tropical

\section{Agroecosystems}

A New Conservation Paradigm

Ivette Perfecto $^{a}$ and John Vandermeer ${ }^{a, b}$

Ann. N.Y. Acad. Sci. 1134: 173-200 (2008).

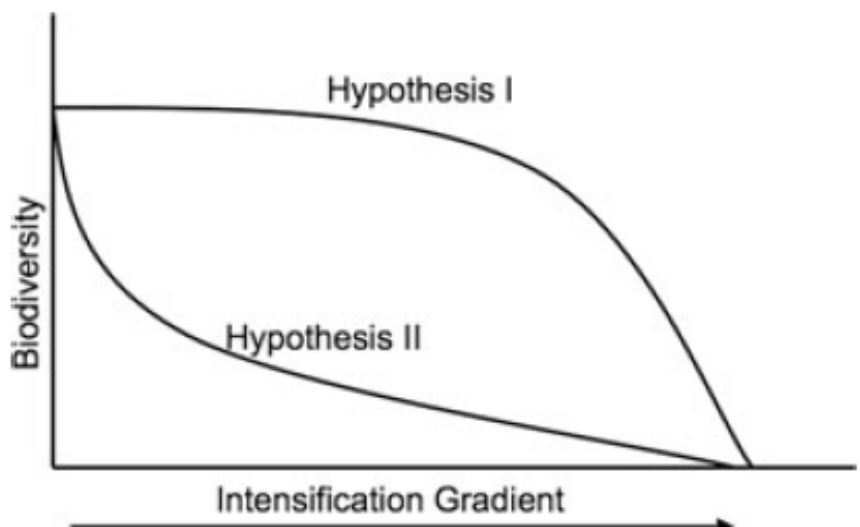

Figure 1. Two hypotheses about the relationship between management intensity and biodiversity.

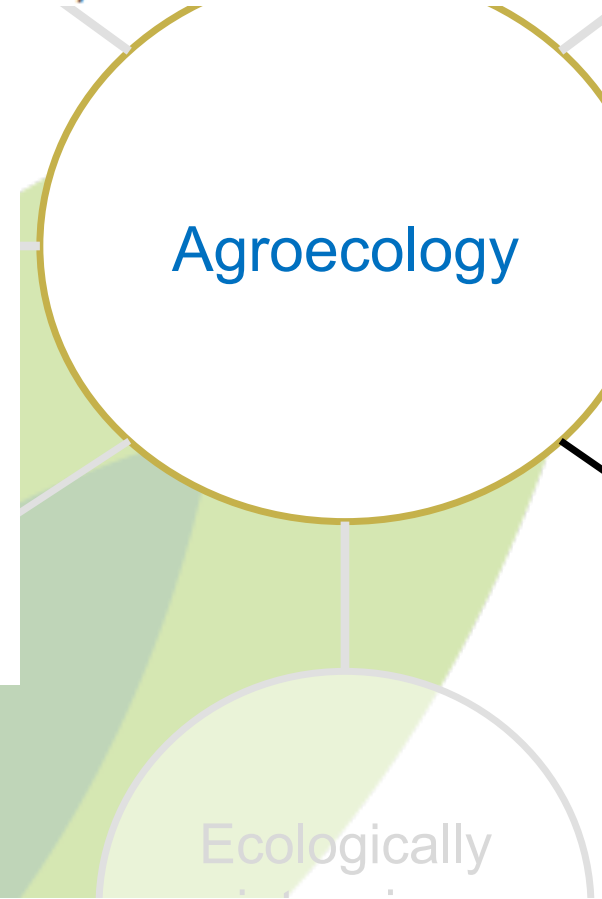

Reconciling Food Production and Biodiversity Conservation: Land Sharing and Land Sparing Compared Ben Phalan et al.

Science 333, 1289 (2011);

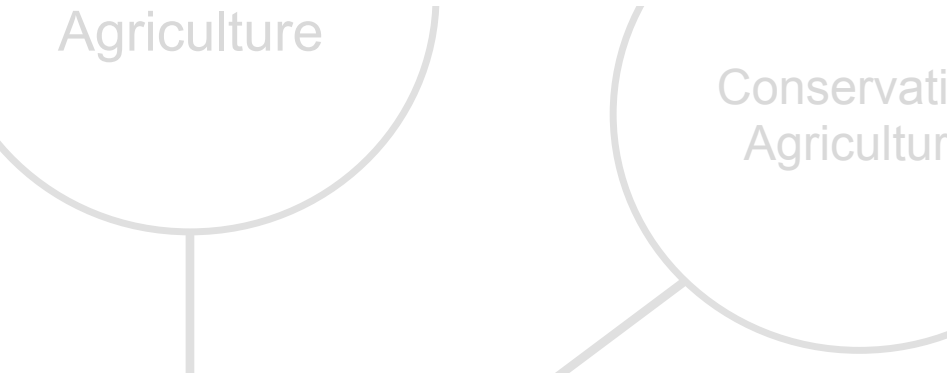
Academy of Sciences.

EcoAgriculture
МIAAAS

\section{Science}

MIAAAS

W.: 10 Jeffrey A. Mcliedy and Sara p, Scher,
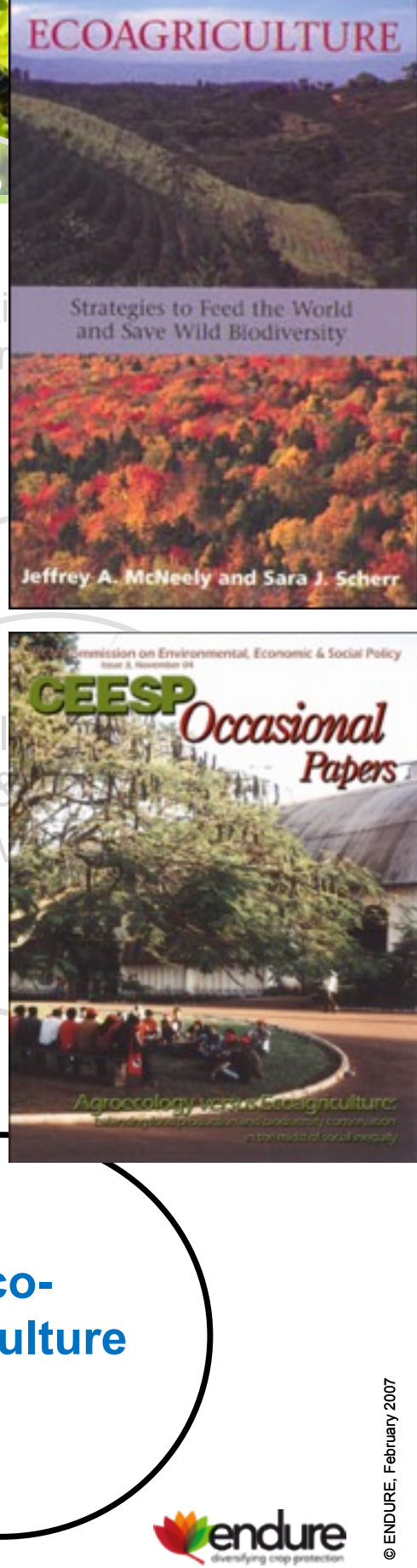


\section{L'agriculture écologiquement intensive :}

nature et défis

Sylvie Bonny Cah Agric, vol. 20, n 6, novembre-décembre 2011 agronomy: Revisiting methods, concepts and knowledge

Thierry Doré, David Makowski, Eric Malézieux, Nathalie Munier-Jolain, Marc Tchamitchian, Pablo Tittonell
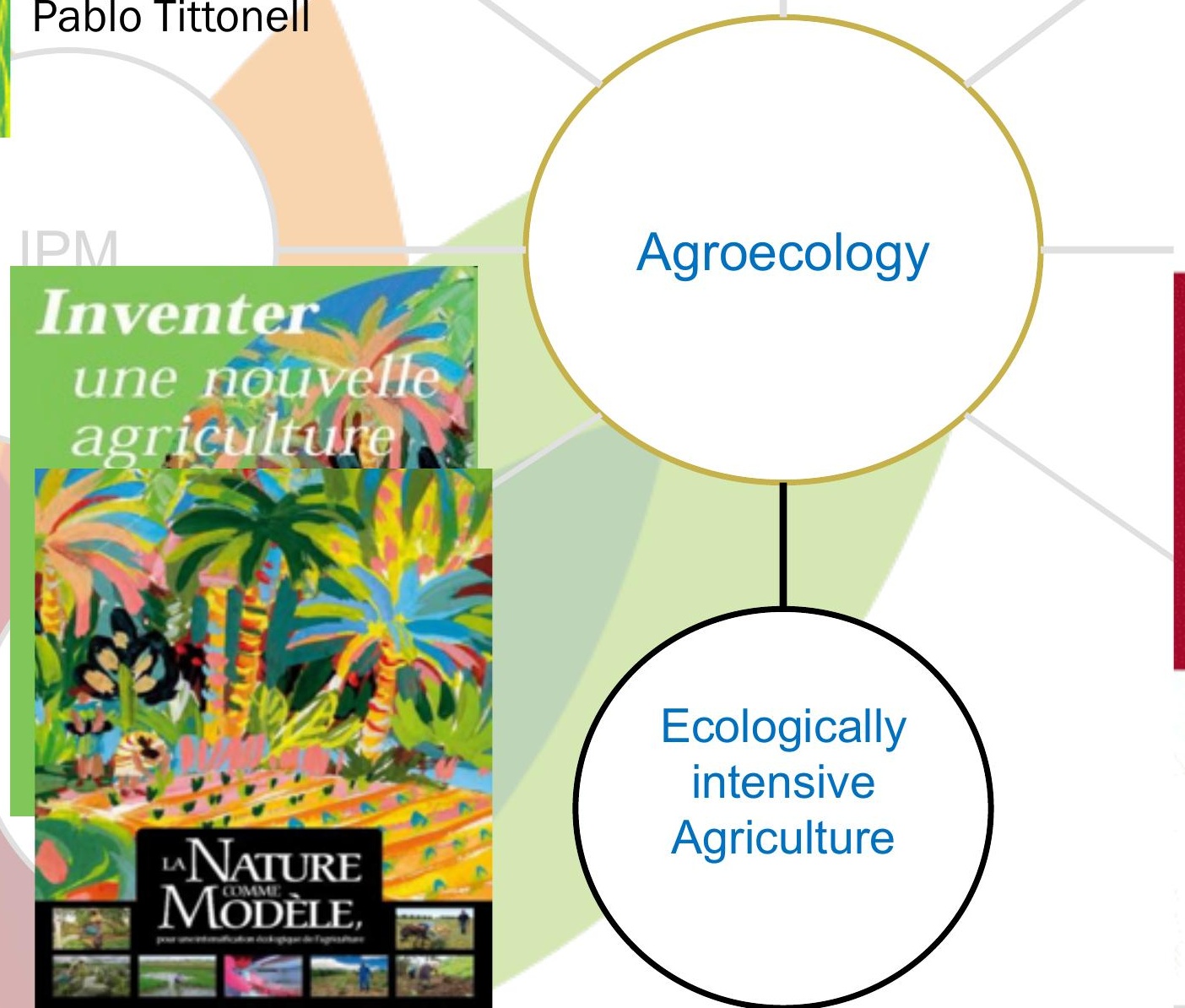

Michel Griffon

Pour des agricultures écologiquement intensives

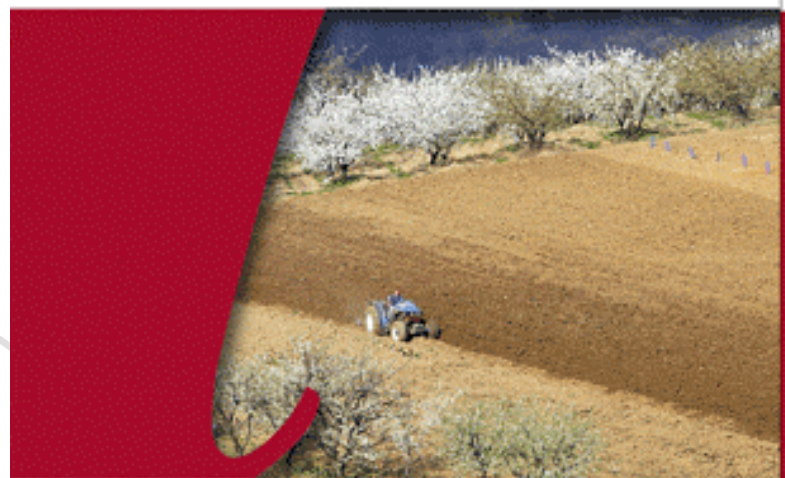

L'agriculture doit effectuer une véritable révolution technologique, et celle-ci doit être accompagnée de politiques agricoles nouveles.

intensive Agriculture 


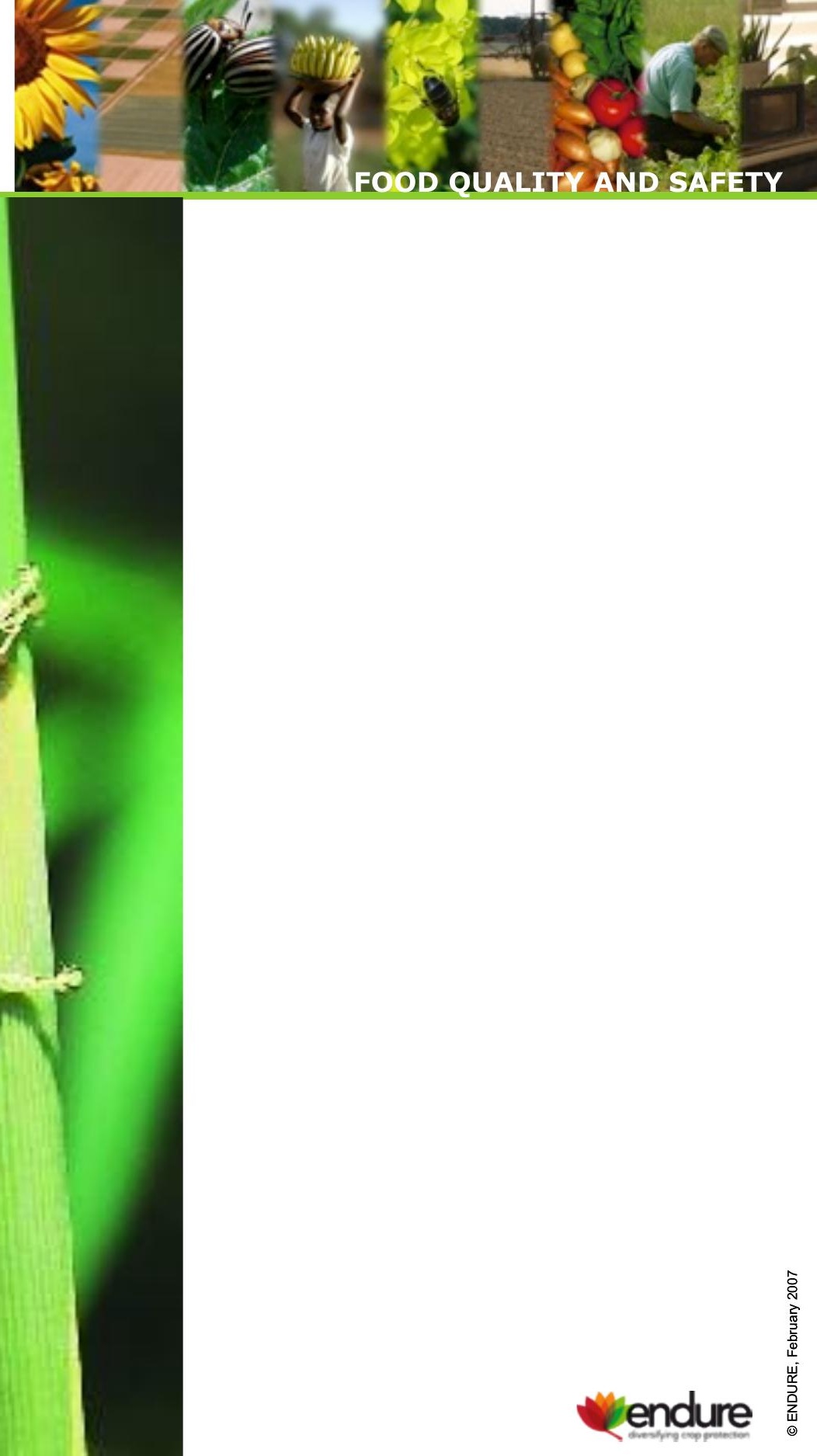

